\title{
Avoiding Animal Testing Under the U.S. Family Smoking Prevention and Tobacco Control Act
}

\author{
Joseph R. Manuppello
}

$\mathbf{N}$ EXT-GENERATION NICOTINE PRODUCTS, including electronic nicotine delivery systems (ENDS), are the focus of the U.S. Food and Drug Administration's (FDA's) contentious May 2016 rule deeming tobacco products to be subject to the Family Smoking Prevention and Tobacco Control Act (FSPTCA) ${ }^{1}$ as well as of its accompanying draft guidance for industry on premarket applications for ENDS. ${ }^{2}$ This timely special issue of Applied In Vitro Toxicology addresses the urgent need for in vitro tests and nonanimal testing strategies to evaluate the toxicity and abuse liability of next-generation nicotine products as compared with other tobacco products on the market. ${ }^{3}$ In addition, there is a need for the investigators developing nonanimal approaches to advocate for their acceptance by regulators. People for the Ethical Treatment of Animals (PETA) has monitored the regulation of tobacco products in the United States since the enactment of the FSPTCA in 2009, submitting comments on rulemaking and guidance and meeting with FDA's Center for Tobacco Products (CTP). Hereunder, we summarize the current U.S. regulatory environment with regard to animal testing and discuss several means by which testing could be reduced.

In the United States, there are currently no requirements for testing tobacco products on animals. Manufacturers are free to use only nonanimal tests to support marketing applications for new products. The FSPTCA states only that FDA's determination as to whether permitting a tobacco product to be marketed would be appropriate for the protection of the public health shall be made on the basis of "well-controlled investigations, which may include one or more clinical investigations." Animal testing is not mentioned.

However, testing tobacco products in animals is not prohibited in the United States, as it is in Belgium, Estonia, Germany, Slovakia, and the United Kingdom. In its 2011 draft guidance on applications for premarket review of new tobacco products, FDA advises manufacturers to evaluate the toxicity, abuse liability, and carcinogenicity of new products "using some combination of in vitro, in vivo, and/or ex vivo studies." 4 In vivo studies are similarly listed among the types of nonclinical studies described in FDA's 2012 draft guidance on modified risk tobacco product (MRTP) applications. As a result, concern remains that manufacturers of next-generation nicotine products will conduct animal tests in support of marketing applications.

More recently, in its deeming rule and guidance documents on tobacco product master files (TPMFs) and premarket applications for ENDS, FDA has offered several means by which the number of tests conducted might be reduced. In its deeming rule, FDA states its intention to issue standards prohibiting the inclusion of characterizing flavors (recognizable flavors other than tobacco) in cigars and indicates that it is also considering standards for ENDS components. Although it is unlikely that such flavors will be entirely prohibited in ENDS, standards could limit exposure to potentially harmful constituents. Such precautionary standards would immediately protect the public health while avoiding animal tests to support the inclusion of these substances in new products. Likewise, FDA issuing standards to limit the temperatures that can be achieved with aerosolizing apparatus may reduce the production of potentially hazardous substances (i.e., carbonyls) by heating e-liquids to high temperatures.

In addition, FDA has stated its intention to open public dockets for substances likely to be used in e-liquids, including carriers, nicotine, colorants, and flavoring agents. Toxicity data submitted on these substances can then be cited in marketing applications. TPMFs are another means by which duplicative testing can be avoided. These files typically contain confidential information that the TPMF owners do not wish to make public. TPMF owners may then authorize other entities to use information in their TPMFs to support submissions to FDA without disclosing that information. Suppliers of components used in ENDS products can reduce animal testing by developing robust TPMFs that include available toxicity data and by authorizing manufacturers to use this information in support of marketing applications for new products.

With regard to animal testing, FDA's draft guidance on ENDS improves upon its previous guidance on premarket review and MRTP applications. FDA stresses its consideration of "valid scientific evidence" in addition to "well-controlled

Regulatory Testing Department, People for the Ethical Treatment of Animals, Norfolk, Virginia.

( ) Joseph R. Manuppello, 2017; Published by Mary Ann Liebert, Inc. This Open Access article is distributed under the terms of the Creative Commons Attribution Noncommercial License (http://creativecommons.org/licenses/by-nc/4.0/) which permits any noncommercial use, distribution, and reproduction in any medium, provided the original author(s) and the source are credited. 
investigations" in determining whether ENDS products are appropriate for the protection of public health. FDA includes guidance on conducting literature reviews and using computational modeling, as well as on bridging of data from one product to another. FDA also discusses in vitro assays that can be used to evaluate the genotoxic potential of ENDS. Notably, FDA encourages applicants to meet with CTP early in the development process to discuss the suitability and acceptability of nonanimal tests.

These are not the only means by which investigators can support the acceptance of in vitro test methods and nonanimal testing strategies by regulators. FDA's guidance documents on preparing marketing applications for tobacco products, including its ENDS guidance, are still in the draft stage, and the public is invited to comment on any guidance at any time. CTP and its Tobacco Products Scientific Advisory Committee frequently host informative public meetings and webinars with additional opportunities to comment and present research results. Cooperation among investigators, industry, advocates, and regulators is needed to ensure that only human-predictive, nonanimal testing approaches are used to test next-generation nicotine products.

\section{Author Disclosure Statement}

No competing financial interests exist.

\section{References}

1. US Food and Drug Administration. Deeming tobacco products to be subject to the Federal Food, Drug, and Cosmetic Act, as amended by the Family Smoking Prevention and Tobacco Control Act; restrictions on the sale and distribution of tobacco products and required warning statements for tobacco products. Fed Reg 2016:81;28973-29106.

2. US Food and Drug Administration. Premarket tobacco product applications for Electronic Nicotine Delivery Systems (ENDS), guidance for industry, draft guidance. 2016. http:// www.fda.gov/TobaccoProducts/Labeling/RulesRegulations Guidance/ucm499351.htm Accessed January 25, 2017.

3. Manuppello JR, Sullivan KM. Toxicity assessment of tobacco products in vitro. Altern Lab Anim 2015:43;39-67.

4. PETA International Science Consortium. Alternative approaches for acute inhalation toxicity testing to address global regulatory and non-regulatory data requirements. 2016. www.piscltd.org.uk/acute-inhalation-toxicity Accessed January 25, 2017.

Address correspondence to: Joseph R. Manuppello Regulatory Testing Department People for the Ethical Treatment of Animals Norfolk, VA 23510

E-mail: josephm@peta.org 